ARTICLE

DOI: $10.1038 / s 41467-018-07379-4$

\title{
The essential genome of the crenarchaeal model Sulfolobus islandicus
}

\author{
Changyi Zhang ${ }^{1,2}$, Alex P.R. Phillips ${ }^{1,2}$, Rebecca L. Wipfler ${ }^{1}$, Gary J. Olsen ${ }^{1,2} \&$ Rachel J. Whitaker ${ }^{1,2}$
}

Sulfolobus islandicus is a model microorganism in the TACK superphylum of the Archaea, a key lineage in the evolutionary history of cells. Here we report a genome-wide identification of the repertoire of genes essential to $S$. islandicus growth in culture. We confirm previous targeted gene knockouts, uncover the non-essentiality of functions assumed to be essential to the Sulfolobus cell, including the proteinaceous S-layer, and highlight essential genes whose functions are yet to be determined. Phyletic distributions illustrate the potential transitions that may have occurred during the evolution of this archaeal microorganism, and highlight sets of genes that may have been associated with each transition. We use this comparative context as a lens to focus future research on archaea-specific uncharacterized essential genes that may provide valuable insights into the evolutionary history of cells.

\footnotetext{
${ }^{1}$ Carl R. Woese Institute for Genomic Biology, University of Illinois at Urbana-Champaign, Urbana 61801 IL, USA. ${ }^{2}$ Department of Microbiology, University of Illinois at Urbana-Champaign, Urbana 61801 IL, United States. These authors contributed equally: Changyi Zhang, Alex P. R. Phillips. Correspondence and requests for materials should be addressed to R.J.W. (email: rwhitaker@life.illinois.edu)
} 
F orty-one years ago, Woese and Fox identified the Archaea as a novel microbial lineage distinct from Bacteria ${ }^{1}$. The same year, Woese and Fox proposed a model of cellular evolution in which early cellular life diverged in two directions, one to the Bacteria and the other to LEACA, the Last Eukaryotic and Archaeal Common Ancestor, which subsequently split forming the Archaea and Eukaryota domains ${ }^{2-4}$. Increases in genome and metagenome sequence data continue to refine this picture, providing reinforcement for many of its key aspects, improving phylogenetic sampling, and providing additional details ${ }^{5-12}$. With the addition of new lineages, the tree of life itself has evolved with great controversy ${ }^{13-17}$. Some of these new analyses suggest that the Thaumarcheota, Aigarchaeota, Crenarchaeota, and Korarchaeota (TACK) lineages of Archaea have the closest relationship to the Eukaryota with cultured representatives $5,6,13,14,18,19$.

Today, the tree of life provides a framework for studying the evolution of cellular complexity. Genomics and metagenomics provide data on the distribution of genes across this tree and in doing so provide an understanding of the origins and evolutionary dynamics of gene sequences. However, phyletic distributions fall short of establishing the functional evolutionary history of the cell since gene presence does not link directly to function. Mapping evolution of today's complex cells involves a comparative approach in which functional cellular systems and the interactions of their constituent components are examined at a molecular level in organisms representing key evolutionary lineages across the tree of life.

As a step in that direction, we take here a genome-wide functional approach to define 441 genes essential to the growth of Sulfolobus islandicus. Sulfolobus, a thermoacidophilic genus from geothermal hot springs, is one of the few organisms within the TACK archaea that can be cultured and is genetically tractable 20,21 , and it is the most developed model for studying the biology of cells in this lineage. We find that the S-layer protein, which is present in most cells in the archaeal domain ${ }^{22}$, is not essential in this model organism. Furthermore, we explore potential stages of evolution of the essential gene repertoire of the archaeal cell.

\section{Results and Discussion}

Identifying essential genes in the genome of $\boldsymbol{S}$. islandicus. We established three independent genome-wide disruption libraries in an agmatine-auxotrophic strain of S. islandicus M.16.4 by using a modified in vitro transposon mutagenesis system derived from Tn5 (Epicentre, USA). The transposable element was comprised of a nutritional marker cassette, SsoargD (arginine decarboxylase derived from Sulfolobus solfataricus P2), flanked by two 19-base pair (bp) inverted repeats (Fig. 1a). After electroporation-mediated transformation of $\mathrm{ArgD}^{-}$cells with the EZ-Tn5 transposome, cells were allowed 10 days of growth on rich media. While valuable information about metabolic and regulatory genes could have been gained by comparing results from different media conditions, we restricted this study to one rich medium to focus on central cellular rather than metabolic functions. Insertion locations were determined via genome tagging and fragmentation ("tagmentation") on colony pools, followed by amplification and sequencing of the junction sites, which were then mapped onto the genome. In all, 89,758 unique insertion events with at least 3 reads each were identified across all three libraries, corresponding to an average of 1 insertion every $29 \mathrm{bp}$ and an average expected 29 insertions in each annotated protein-coding gene (see Methods; Supplementary Table 1 contains colony, insertion, and read counts for each library while all insertion locations can be found in Supplementary Data 1).
Essential genes were predicted to be significantly underrepresented in the insertion locations extracted from the transposon mutagenesis and sequencing data (Tn-seq). It is important to note that this may make them indistinguishable from genes that are not strictly essential for growth but instead cause a severe growth defect, and thus our definition of "essential" extends to these genes too. To determine the statistical separation between essential and non-essential genes, we used a combination of two programs: ESSENTIALS ${ }^{23}$ and Tn-Seq Explorer ${ }^{24}$. Both methods report essential gene candidates by separating essential and non-essential genes into a bimodal distribution of scores. ESSENTIALS does so by calculating a log ratio of observed and expected reads in each gene $\left(\log _{2} \mathrm{FC}\right)$, while Tn-Seq Explorer uses a sliding window approach to examine the absolute number of insertions in and around genes and calculates an Essentiality Index (EI) for each. The former tends to underestimate the number of essential genes, while the latter tends to overestimate $^{24}$. Four hundred and forty-five genes lie within the suggested range for both methods $\left(\log _{2} \mathrm{FC} \leq-5.1\right.$ and $\left.\mathrm{EI}<4\right)$, leaving 175 genes within only one range or "unassigned" as essential or non-essential. Fourteen protein-encoding genes could not be assigned a $\log _{2} \mathrm{FC}$ score due to the presence of identical sequence elsewhere in the genome (score "NA" in Supplementary Data 2). The remaining 2100 protein-coding genes are likely nonessential for growth under these conditions (Fig. $1 \mathrm{~b}$ and Supplementary Data 2). Three genes identified as essential through automated methods were additionally removed because misplaced multiply mapped reads falsely reduced read count (M164_0862, M164_1012, and M164_1867; see Supplementary Table 2). Assignments of all genes to categories with their scores for each method are listed in Supplementary Data 2.

Genetic confirmation of essential gene criteria. To support our informatic essentiality/non-essentiality criteria, 129 genes were compared with gene knockout studies performed in our model S. islandicus M.16.4 and another two genetically tractable $S$. islandicus strains: RYE15A and LAL 14/1 (Supplementary Table 3). We were unable to acquire knockouts for 42 of the 45 predicted essential genes in this set. Two exceptions, topR2 (M164_1245) and apt (M164_0158), were identified to have significant growth defects on plates once they were knocked out (Supplementary Fig. $1 \mathrm{c}$ and $2 \mathrm{a}$ and ref. ${ }^{25}$ ), likely resulting in their under-representation in our transposon library. The third, $c d v B 3$ (M164_1510), a paralog of $c d v B$, may be incorrectly called essential in our Tn-seq analysis. We can readily obtain $c d v B 3$ disruption mutants (Supplementary Fig. 3b) and the growth of a $c d v B 3$ mutant strain is indistinguishable from the wild-type strain (data not shown), thus this gene was removed from the essential gene list. An explanation of why this gene is mischaracterized would require further investigation, but it is possible that, because the score distributions for essential and non-essential genes overlap, this gene was simply not hit enough times to achieve significance. This could be true for a small number of other genes as well and is a fundamental limitation of Tn-seq.

To further investigate our automated assignments, we screened eight "unassigned" genes in S. islandicus M.16.4 that were called essential by one method or the other but not both. We were unable to obtain mutants for six of them. Of these, five genes, i.e., lig (M164_1953), priL (M164_1568), priX (M164_1652), rnhII (M164_0197), and $t f s 2$ (M164_1524), were called essential via EI but not $\log _{2}$ FC, while thrS1 (M164_0290) was called essential based on $\log _{2} \mathrm{FC}$ but not EI. In contrast, knockouts of the two "unassigned" genes called essential by EI but not $\log _{2} \mathrm{FC}, u d g 4$ (M164_0085), encoding uracil-DNA glycosylase family 4, and 
a

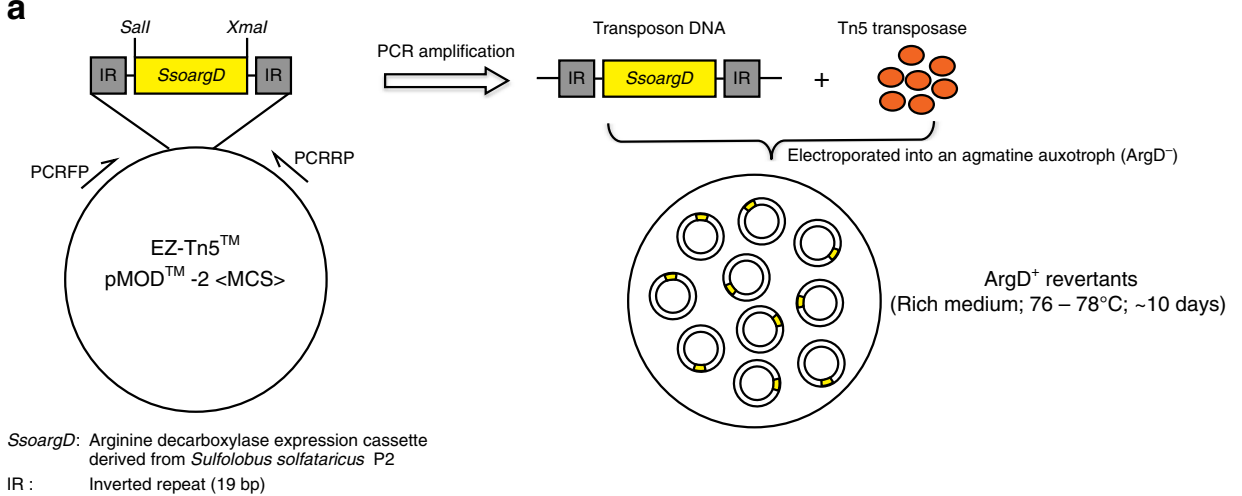

IR: Inverted repeat (19 bp)

b

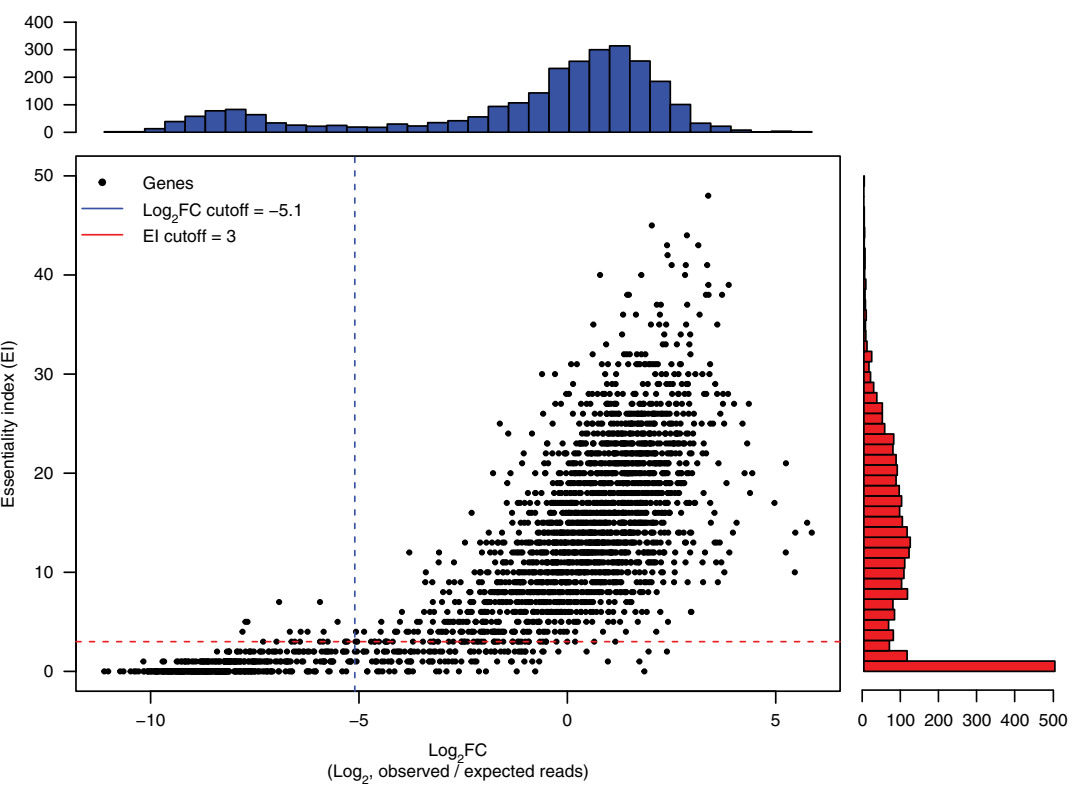

Fig. 1 Defining the essential genes in S. islandicus M.16.4. a Schematic overview of the genome-wide transposon mutagenesis strategy. $\mathbf{b}$ Evaluation of gene essentiality by two computational programs: ESSENTIALS ${ }^{23}$ and Tn-Seq-Explorer ${ }^{24}$. Points indicate individual genes plotted according to the scores returned by each program. Histograms indicate the number of genes of a particular score, and the dotted lines indicate the recommended cutoffs returned by each program as the local minimum between the essential and non-essential score distributions. Essential genes meet both criteria (lower-left quadrant). The protein-coding genes that only met the ESSENTIALS or Tn-Seq-Explorer criteria were deemed as "unassigned candidates" leaving the rest as likely non-essential to S. islandicus M.16.4 growth under these conditions. A complete list of the $\log _{2} \mathrm{FC}$ and El for the S. islandicus M.16.4 genes from the combined mutant libraries are provided in Supplementary Data 2

rpo8 (M164_1872), encoding a subunit of RNA polymerase, were obtained after an extended 14 days incubation of transformation plates, again consistent with a severe growth defect (Supplementary Fig. 2b, c and 3b). This suggests the presence of false negatives and a stronger bias to underestimate than overestimate the true number of essential genes. Because not all genes in the unassigned categories were genetically tested, we conservatively excluded all unassigned genes from the essential gene list. By contrast, knockouts for all 76 non-essential genes tested were successfully obtained and verified by PCR analysis (Supplementary Table 3 and Supplementary Fig. $3 \mathrm{a}$ and $3 \mathrm{~b}$ ). These include hjm/hel308a (M164_0269), cdvB1 (M164_1700), topR1 (M164_1732), and three DExD/H-box family helicase genes (M164_0809, M164_2103, and M164_2020), the homologs of which were previously thought to be essential in a related strain S. islandicus Rey15A $\mathrm{A}^{26-29}$ (Supplementary Table 3 and Supplementary Fig. 3b). Taken together, these experimental results supported the overall validity of our computational approaches for conservatively classifying putative gene essentiality.
Essential gene repertoire. The functional repertoire of the predicted essential, unassigned, and non-essential genes of S. islandicus is shown in Fig. 2. With the above adjustments, the size of this essential genome (441 genes) is close in size to that observed for other bacteria and archaea ${ }^{30}$. For example, $\sim 526$ genes are required for growth in Methanococcus maripaludis S2 ${ }^{31}$ and 473 genes within the engineered Mycoplasma mycoides JCVI Syn3.0 minimal bacterial cell ${ }^{32}$. The proportion of different functional categories represented in this set (as defined by archaeal clusters of orthologous genes ${ }^{12}$ (arCOGs)) are also similar to that observed in other studies ${ }^{31,32}$ (Fig. 2), with the largest fraction of genes $(178, \sim 40 \%)$ representing information processing (translation, transcription, and DNA replication/ recombination/repair) and $76(\sim 17 \%)$ either classified as "function unknown" or "general functional prediction only." The latter two categories are hereby collectively referred to as "poorly characterized." Descriptions of the specific essential components found in central information processing and the cell cycle, as well as central carbon metabolism, are detailed in Supplementary 


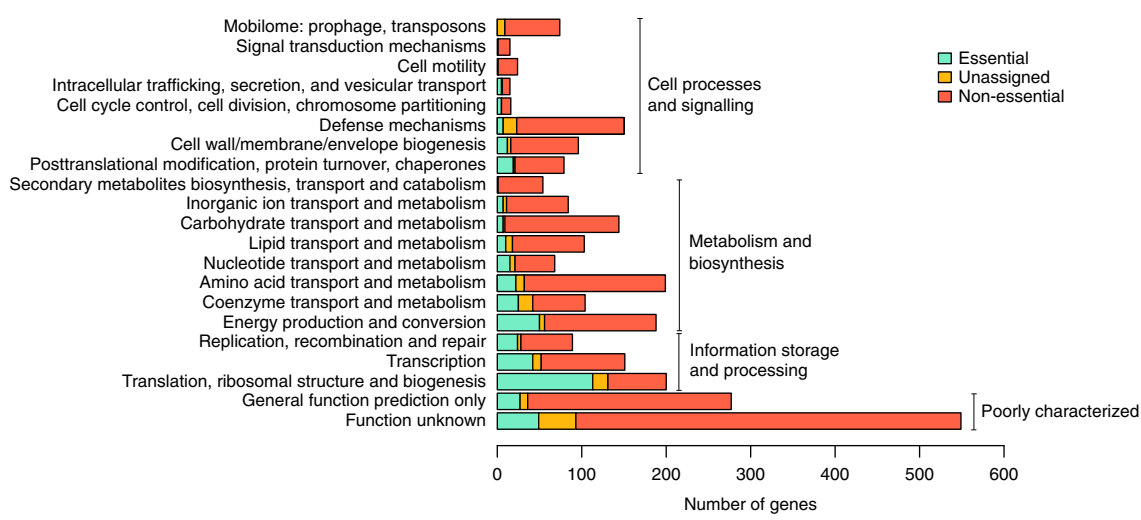

Fig. 2 arCOG category and essentiality criteria for protein-coding genes in S. islandicus M.16.4. Functional distribution of essential, non-essential, and unassigned genes via arCOG category. Essentiality criteria based on cutoffs in Fig. 1b

Notes 1-5. We highlight only a few interesting and novel observations below.

S-layer is non-essential in S. islandicus. Our essential gene predictions include several surprising findings. First, SlaA (M164_1763) and SlaB (M164_1762), the two known components of the surface layer (S-layer) of Sulfolobus cells ${ }^{33}$ were shown to be non-essential. SlaA is the dominant component of the S-layer that forms a quasi-crystalline matrix outside the cell membrane ${ }^{34}$. Current models suggest a "stalk-and-cap" structure in which the C-terminal-transmembrane-helix-domain-containing SlaB projects from the cell membrane and anchors SlaA to the cell membrane $22,35,36$. The cellular function of the Sulfolobus Slayer is unknown but is believed to provide resistance to osmotic stress and contribute to cell morphology $y^{33}$. S-layer-deficient mutants have never been successfully cultivated before in any archaeal species, therefore it was assumed to be essential.

To confirm the non-essentiality of the S-layer genes, we constructed in-frame deletion mutants of sla $A$, sla $B$, and sla $A B$ via a MID (marker insertion and unmarked target gene deletion) recombination strategy ${ }^{37}$. PCR amplification with two primer sets, which bind the flanking and internal region of S-layer genes, respectively (Fig. 3a), confirmed the successful deletion of slaA, $s l a B$, and $s l a A B$ from the chromosome of the genetic host RJW004 (wild type) (Fig. 3b). We next tested for absence of the S-layer proteins in growing cells. Isolation of a white precipitant, described as the S-layer previously ${ }^{38}$, was possible only in the wild type and to a much lesser extent in the $\Delta$ slaB mutant strain (Supplementary Fig. 4a and 4b). Transmission electron microscopy (TEM) analysis confirmed this extracted protein precipitate from both wild type and $\triangle$ slaB mutant strains formed crystalline lattice structures (Supplementary Fig. 4c). Finally, we tested the mutant phenotypes by comparing their growth profiles with wild type in a standard laboratory condition ( $\mathrm{pH} \mathrm{3.3,76}{ }^{\circ} \mathrm{C}$ ). As shown in Fig. $3 \mathrm{c}$, cells lacking the Slayer protein lattice SlaA (including slaA and sla $A B$ mutants) are viable but have a measurable growth defect. This confirms the non-essentiality of the S-layer lattice in S. islandicus. The deletion of $s l a B$ alone had no significant impact on the growth rate in comparison with that of wild type (Fig. 3c). For a complete knockout of all potential S-layer components, we successfully created a viable triple knockout of $s l a A$, slaB, and a paralog of SlaB encoded by M164_1049 (42\% coverage, 53\% amino acid identity via BLAST), demonstrating non-essentiality of all S-layer components together in S. islandicus (Supplementary Fig. 5).

We performed thin-section TEM analyses of the RJW004 (wild type) and S-layer gene knockout strains. The thin-section micrographs of wild-type cells clearly revealed that the S-layer was separated from the cytoplasmic membrane by a quasiperiplasmic space (Fig. 4a, e), in agreement with previous studies in Sulfolobus acidocaldarius ${ }^{39}$ and Sulfolobus shibatae ${ }^{34}$. The S-layer in the wild type was observed as a distinct dark band on the outermost edge of the cell, and the quasi-periplasmic space was seen as a light gray band between the outermost band and the cell membrane (Fig. 4a, e). However, the dark, outermost layer surrounding the cell was not observed in the $\triangle \operatorname{slaA}$ or $\triangle$ sla $A B$ mutant cells (Fig. $4 \mathrm{~b}, \mathrm{~d}, \mathrm{f}, \mathrm{h}$ ), confirming that SlaA contributes to the formation of the outermost layer. Additionally, the cell surface appeared diffuse in the $\Delta$ slaA mutant cell, which was attributed to the periodic extensions of membrane proteins, likely including the SlaB protein, and/or their extensive $\mathrm{N}$ glycosylation $^{22}$. In the $\Delta$ sla $B$ mutant cell, a smooth outermost layer similar to the SlaA layer in wild-type cells was observed; however, it appears to be discontinuous around the cell membrane (Fig. $4 \mathrm{c}, \mathrm{g}$ ). The partial lattice of SlaA in the $\Delta$ slaB mutant may be anchored by other membrane proteins even in the absence of SlaB, including the aforementioned M164_1049. Together these images suggest that additional components may contribute to the non-essential Sulfolobus S-layer.

Incomplete complementarity of reverse gyrase. As an additional surprise from our genome-wide essential gene identification, we found incomplete complementarity between two copies of the reverse gyrase in S. islandicus M.16.4. Unlike Euryarchaota and most extremely thermophilic bacteria, Crenarchaeota possess two copies of reverse gyrase ${ }^{40,41}$, both believed to be essential for growth ${ }^{26,42}$. Tn-seq analysis indicated that the topR1 (M164_1732) was non-essential, which was confirmed by a successful disruption (Supplementary Fig. 1a and 1b). Interestingly, as mentioned above, topR2 (M164_1245) was called essential but we could obtain topR2 disruption mutants (Supplementary Fig. 1a and 1c) if we prolonged the incubation time (up to 14-20 days) of transformation plates in gene knockout experiments. These observations suggest that topR2 plays a more important role than topR1 in Sulfolobus cell survival at optimal temperature.

Lethal deletion mutants. Tn-seq also uncovered genes that may not be essential to growth but instead are toxic when disrupted. Among them, arCOG analysis predicts that M164_0131, M164_0217, M164_0268, M164_2076, M164_1728, and M164_1060 are antitoxin-encoding genes. We reason that inactivation of these antitoxin genes might cause overproduction of toxins and then trigger cell death. This finding suggests that associated toxins are constitutively expressed in our laboratory conditions. Interestingly, unlike most of the family II (VapBC) and family HEPN-NT toxin/antitoxin gene pairs in S. islandicus 
a
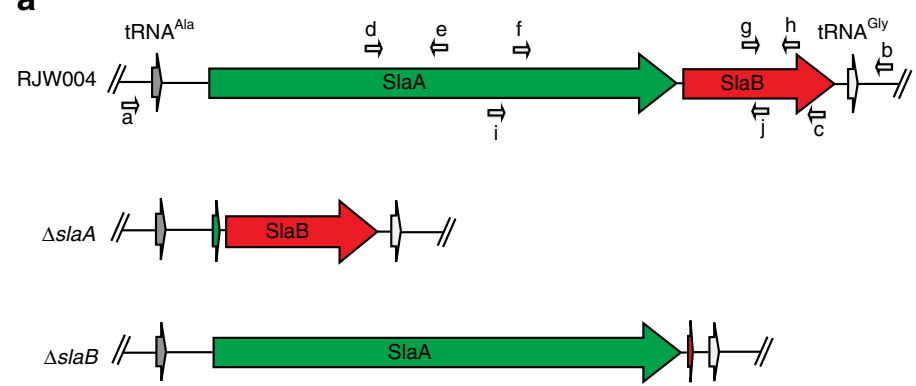

$\triangle$ slaAB //

C

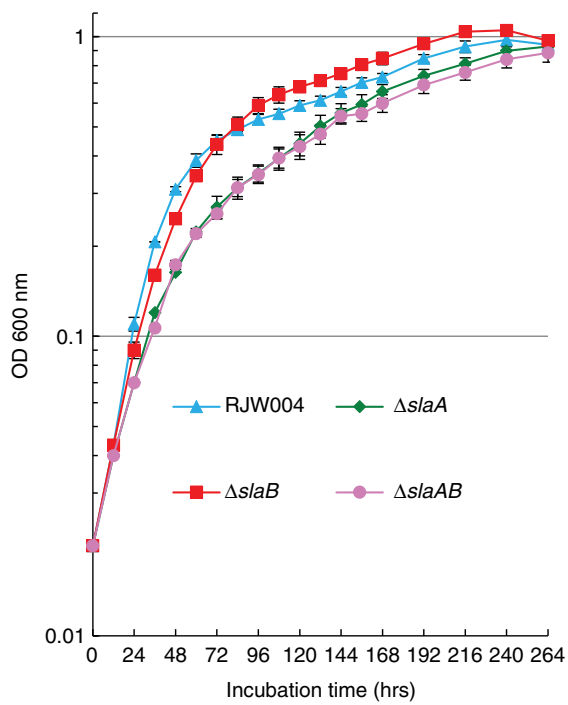

b

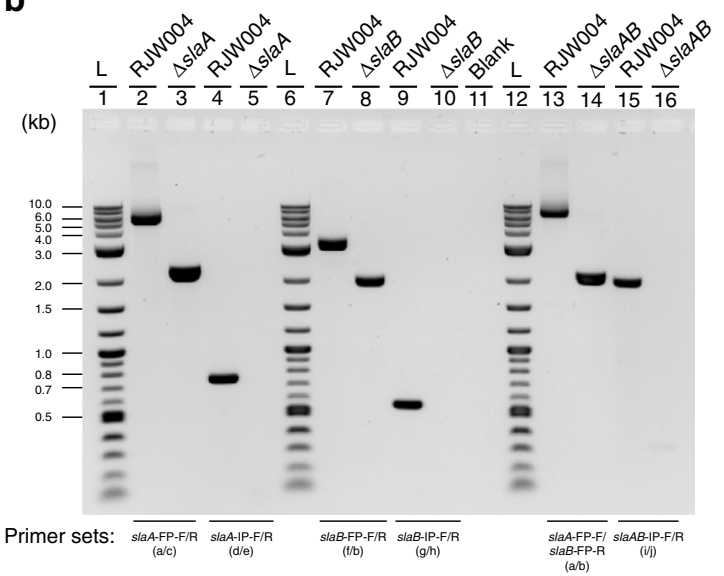

ig. 3 S-layer genes are not essential for the S. islandicus cell survival. a Genomic context of S-layer genes in the genetic host and mutant strains. Relative positions of primers used to confirm S-layer gene deletions are labelled with small arrows. b PCR verification of $\Delta s / a A, \Delta s l a B$, and $\Delta$ slaAB mutants with two primer sets, which bind the flanking and internal regions of S-layer genes, respectively. Expected sizes of amplicons can be found in Supplementary Table 8 . $\mathrm{L}$ (lanes 1, 6, and 12) indicates the 2-Log DNA Ladder (NEB, USA). Blank (lane 11) denotes that no sample was loaded in the well. c Growth profiles of RJW004 (wild type), $\Delta s l a A, \Delta s l a B$, and $\Delta s l a A B$ mutant strains. Wild-type and S-layer gene knockout strains were cultivated at $\mathrm{pH} 3.3,76^{\circ} \mathrm{C}$ for 11 days in DY liquid medium supplemented with uracil and agmatine without shaking. Cell culture growth was monitored by optical density measurements at $600 \mathrm{~nm}$ every 12 or $24 \mathrm{~h}$. Error bars represented standard deviations from three independent experiments

M.16.4 (Supplementary Data 3), partners (toxin genes) adjacent to these predicted antitoxin genes (with the exception of M164_1060; see Supplementary Fig. 6) were not observed. This indicates that VapB-VapC or HEPN-NT do not always correspond to their neighbors and some gene pairs might have exchanged counterparts. The Tn-seq-based analyses also classified cas5 (M164_0911), a part of the Cascade (CRISPR-associated complex for antiviral defense) complex ${ }^{43}$, as essential. Consistent with this assignment, disruption of cas 5 by replacing it with the StoargD marker cassette via homologous recombination failed after repeated attempts. However, the entire Type-IA module of CRISPR-Cas system, consisting of eight genes with cas 5 included, could be deleted from the S. islandicus M.16.4 chromosome with no detectable effect on cell growth (data not shown). One possible explanation is that, in the absence of cas5, the Cascade complex becomes misfolded and thus toxic for the cells, but future studies are needed to confirm this interpretation.

Shared essential genes. To establish how this essential gene set compares with those found in other organisms, we retrieved sets of essential genes from the database of essential genes ${ }^{30,44}$ in eight model organisms that span the tree of life $\mathrm{e}^{31,45-49}$, including the minimal genes set in the JCVI Syn 3.0 Mycoplasma mycoides genome $^{32}$ (Fig. 5, Supplementary Data 4). We find that $242 \mathrm{~S}$. islandicus essential genes are essential in at least 1 other organism we surveyed, while 199 essential genes are uniquely essential in $S$. islandicus. Eighty-nine genes are essential in representatives of all 3 domains, 78 of which are also essential in Syn 3.0 (Supplementary Data 4). As shown in Fig. 5, comparisons of shared essential genes support the shared cellular systems between the archaeal and eukaryotic domains. More total S. islandicus essential gene orthologs are shared with archaea and eukaryotes (gray in Fig. 5), and more of these shared orthologs are essential (colors), than are shared between $S$. islandicus and the bacteria we use for comparison. The highest number of shared essential genes (187) is between $S$. islandicus and M. maripaludis S2 ${ }^{31}$, an organism from the euryarchaeal lineage of the archaeal domain (Table 1). The large size of the essential gene set shared between Sulfolobus and Methanococcus, in spite of their widely different habitats and life styles, reinforces the fundamental nature of Archaea as a distinct cell type $\mathrm{e}^{50}$.

Phyletic distributions of essential genes. To investigate the broader phyletic distributions of S. islandicus essential genes, we 
a

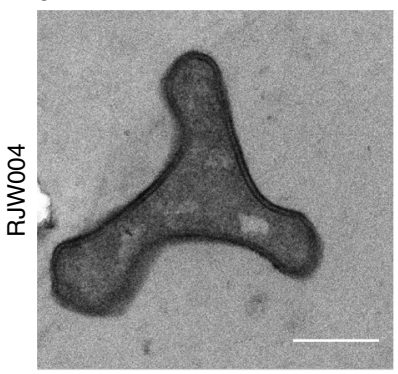

b

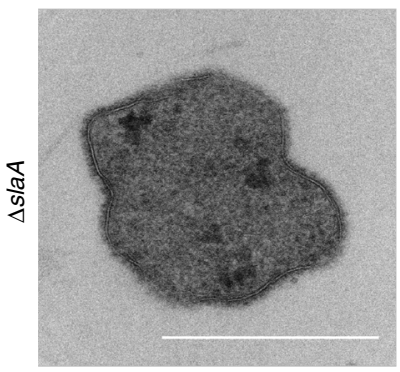

c

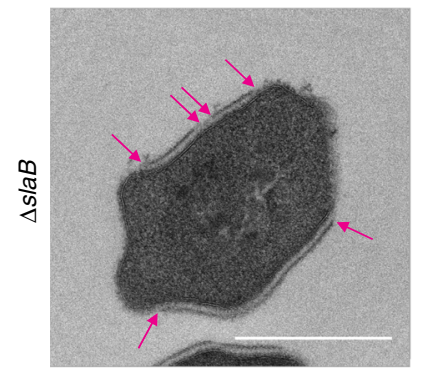

d

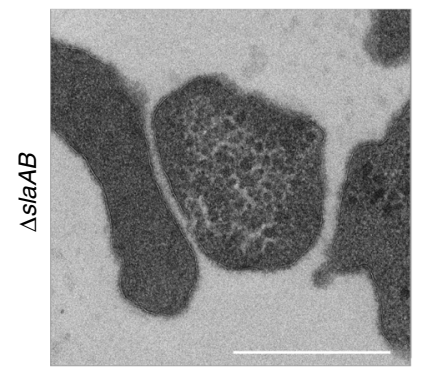

e

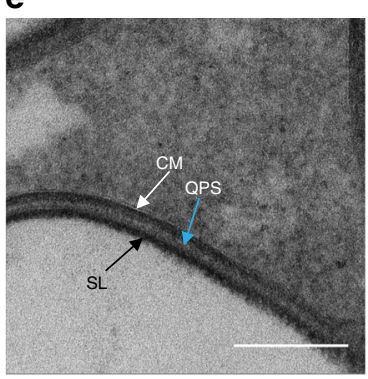

f

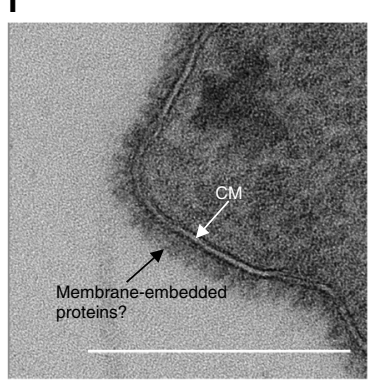

$\mathbf{g}$

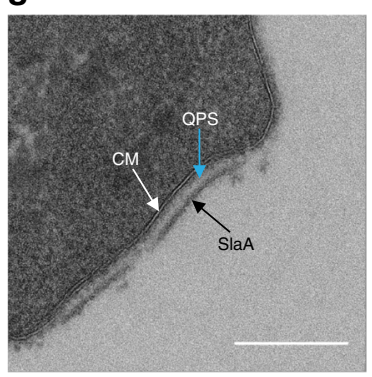

h

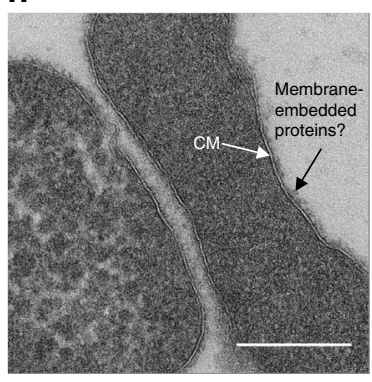

Fig. 4 Thin-section TEM analysis of the wild-type and S-layer gene knockout strains. a-d Representative TEM micrographs of thin-sectioned cells of the wild-type, $\Delta s l a A, \Delta s l a B$, and $\Delta$ slaAB mutant strains, respectively. Images $\mathbf{e}-\mathbf{h}$ are closeups of images $\mathbf{a}-\mathbf{d}$, respectively. Magenta arrows indicate the breaking points of S-layer. CM cytoplasmic membrane, SL surface layer, QPS quasi-periplasmic space, SlaA surface layer protein A. Scale bars, $500 \mathrm{~nm}(\mathbf{a}-\mathbf{d})$ and $200 \mathrm{~nm}(\mathbf{e - h})$

used assignments from the evolutionary genealogy of genes: Nonsupervised Orthologous Groups (eggNOG) database ${ }^{51}$ (see Methods) to map the presence and absence of putative essential gene orthologs from 168 complete genomes representing major clades in all 3 domains based on a previously published analysis ${ }^{6}$ (Supplementary Data 5 and Supplementary Data 6). Figure 6 graphically shows the $S$. islandicus essential genes shared in other genomes in a set of hierarchical clusters based on Euclidean distance. From this Fig. 4, primary transitions emerge in the evolution of the contemporary S. islandicus essential genome. The number of genes in phyletic groups (Table 2) is significantly

different from random sampling among phyletic categories (Supplementary Table 4). Similarly ranked distributions are seen in two additional datasets: (1) all genomes in the eggNOG database subsampled to have equal representation in each domain and (2) all genomes in the eggNOG database for which assignments are available. These data are supported by parsimony analysis with bootstrap support for the grouping of each of the three major domains (Supplementary Figs. 7 and 8). Together these data support four primary stages in the evolution of the contemporary $S$. islandicus cells and allow us to assign specific essential genes to these potential transitions in the evolution of the cell.

The highest number of essential genes are shared broadly across the tree of life (Universal in Table 2), supporting the early evolution of the majority of essential gene functions in the contemporary archaeal cell. Most of these have putative functional assignments in information processing, particularly translation and transcription (Supplementary Data 7). Many previous studies have reported the evolutionary conservation of information processing components going back to the Last Universal Common Ancestor (LUCA) using computational methods $^{7-9,11,52}$. We find that in all studies the majority of conserved orthologous gene sets that we could interrogate in this system are essential (Supplementary Table 5 and Supplementary Data 8). Of the 200 metabolic COGs identified in the S. islandicus genome from a recent estimate of the LUCA gene set ${ }^{10}$, only 19 were found to be essential (Supplementary Data 8). This is expected, due to our use of rich medium. The first phase of the cell contains the universal set of genes with conserved cellular components that are likely to have evolved early in evolutionary history remain essential components of the contemporary $S$. islandicus genome today.

The next largest category of essential genes is found between Sulfolobus and other organisms in the Eukarya/Archaea (EA) domains (Table 2). These genes are largely involved in core information processing functions and support the shared evolutionary ancestry of the Archaea and Eukarya after their divergence from Bacteria. Only one gene in this category is poorly characterized: M164_0237, a homolog to eukaryotic zpr1. zpr1 is a gene essential for transcription and cell cycle progression in fungal and mammalian cells ${ }^{53-56}$ and has recently been reported as a regulator of circadian rhythm in plants ${ }^{57}$. Though it has been noted that this gene is exclusively shared in EA ${ }^{56}$, it remains uncharacterized in the Archaea outside of our results recognizing its essentiality in Sulfolobus (Supplementary Table 6).

Fifty-five essential genes belong to NOGs that are shared by organisms in the archaeal domain (Table 2). Functional assignments of the archaeal-specific genes represent a diversity of functions split between core functions (translation, transcription, and replication) and peripheral functions, such as transport, defense (including all the above-mentioned predicted antitoxin genes), and metabolism. Archaea-specific DNA replication/ recombination/repair genes are nurA and gins15, while genes in arCOG category "transcription (K)" are largely transcription factors and do not represent core RNA polymerase functionality like the EA genes mentioned above. Fourteen of the archaealspecific genes are poorly characterized (Table 2), 9 of which are also essential in M. maripaludis S2 (Supplementary Data 4). In an evolutionary context, this set of poorly characterized, but essential, archaea-specific genes are key targets for future molecular characterization since they likely highlight the unique biology of archaeal cells. We also show that the majority of $S$. islandicus genes are conserved in evolutionary history through the archaeal domain.

The final set of essential genes are specific or largely specific to the Sulfolobales, most of which have uncharacterized functions 


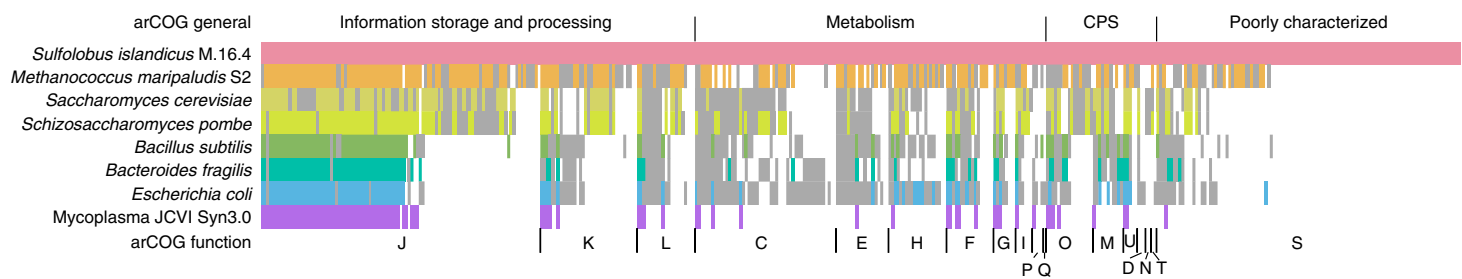

Fig. 5 Shared essential genes across the three domains of life. Heatmap shows the presence of essential (colored) or non-essential (gray) shared NOGs compared with the S. islandicus M.16.4 essential genome. Single-letter codes for functional categories are as follows: J, translation, ribosomal structure, and biogenesis; $\mathrm{K}$, transcription; L, DNA replication, recombination, and repair; $\mathrm{C}$, energy production and conversion; $\mathrm{E}$, amino acid transport and metabolism; $\mathrm{H}$, coenzyme transport and metabolism; F, nucleotide transport and metabolism; G, carbohydrate transport and metabolism; I, lipid transport and metabolism; $\mathrm{P}$, inorganic ion transport and metabolism; $\mathrm{Q}$, secondary metabolite biosynthesis, transport, and catabolism; O, posttranslational modification, protein turnover, chaperone functions; $\mathrm{M}$, cell wall/membrane/envelope biogenesis; $\mathrm{U}$, intracellular trafficking, secretion, and vesicular transport; $\mathrm{D}$, cell cycle control and mitosis; $\mathrm{N}$, cell motility; $\mathrm{T}$, signal transduction; $\mathrm{S}$, function unknown. CPS, cellular processes and signaling

Table 1 Number of $S$. islandicus essential genes shared with seven model organisms

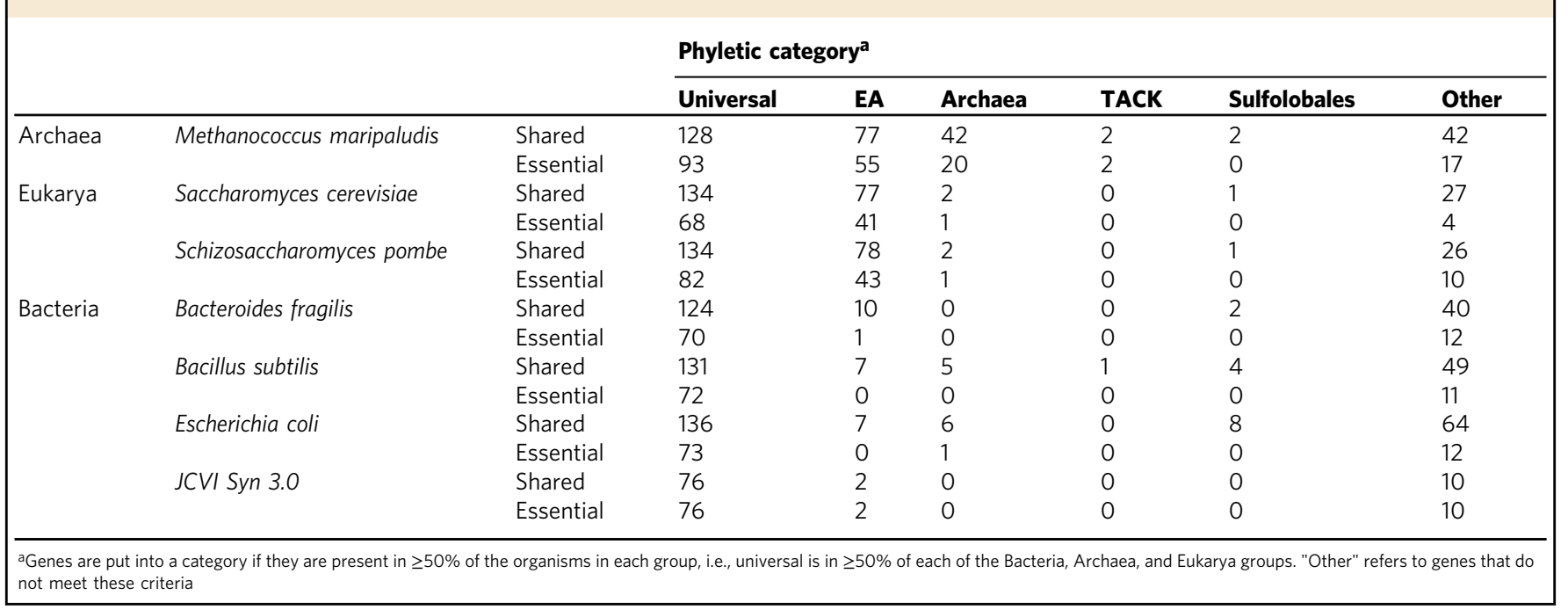

(Table 2). The essentiality of these genes and whether they fit into central cellular functions as non-orthologous gene replacements or peripheral ones are important subjects of future work. This set of genes, unique to this lineage, may represent environmental adaptations. The fact that they are poorly characterized attests to the need for further study even in this model archaeon.

The key next steps toward comparative cell biology will be understanding the functional interactions among essential genes so that new gene inventions, non-orthologous gene transfers, and/or loss of specific functions can be identified. From the unique perspective of the TACK archaea, this work provides a list of genes whose future molecular and systems characterization are likely to provide further understanding for evolutionary steps in the Archaea.

In conclusion, this is the first comprehensive genome-wide study of essential gene content in a model crenarchaeon. Our profile of $S$. islandicus essential genes uncovers several surprising findings, most notably the non-essentiality of the Sulfolobus Slayer. Comparative phyletic patterns provide a perspective on the stages of evolution of the contemporary $S$. islandicus, its shared ancestry with the eukaryotes, and the key components that define its uniqueness as an archaeal cell.

\section{Methods}

Strains and culture conditions. The complete list of strains and plasmids used in this study is shown in Supplementary Table 7. All S. islandicus strains were routinely grown aerobically at $76-78^{\circ} \mathrm{C}$ and $\mathrm{pH} 3.3$ without shaking in basal salt medium ${ }^{37}$ containing $0.2 \%$ [wt $\left./ \mathrm{vol}\right]$ dextrin (Sigma-Aldrich, USA) and $0.1 \%$ [wt/vol] tryptone (BD Biosciences, USA) (the medium is hereafter named as DY). When required, agmatine, uracil, and 5-FOA were added to a final concentration of 50,20 , and $50 \mu \mathrm{g} / \mathrm{ml}$ respectively. For solid plates, $2 \times \mathrm{DY}$ medium was supplemented with $20 \mathrm{mM} \mathrm{MgSO}$ and $7 \mathrm{mM} \mathrm{CaCl} \cdot 2 \mathrm{H}_{2} \mathrm{O}$ and mixed with $1.4 \%$ gelrite (Sigma-Aldrich, USA) with a ratio of 1:1 [vol/vol]. Plates were put into sealed bags and generally incubated for $10-14$ days at $76-78^{\circ} \mathrm{C}$. Cell culture growth was monitored by optical density measurements at $600 \mathrm{~nm}$ using a portable cell density meter (CO8000, WPA, Cambridge, UK).

Construction of S. islandicus transposon mutant library. The 755-bp argD gene cassette (SsoargD) was PCR-amplified from the genomic DNA of S. solfataricus P2 using the primer set SsoargD-F1/R1, introducing the SalI and XmalI sites, respectively. The resultant PCR products were digested with SalI/Xmal and then cloned into the EZ-Tn $5^{\mathrm{TM}} \mathrm{pMOD}^{\mathrm{TM}}-2<\mathrm{MCS}>$ Transposon Construction Vector (Epicentre, USA) in the corresponding sites, generating pT-SsoargD. The Tn5 $<$ SsoargD $>$ transposon DNA was prepared by PCR amplification from linearized pT-SsoargD with 5'phosphorylated primers PCRFP/PCRRP. The PCR products, consisting of a nutritional marker flanked by a 19-bp inverted repeat (Mosaic Ends, $\mathrm{ME}$ ), were purified and highly concentrated using the DNA Clean \& Concentrator $^{\mathrm{TM}_{-}}$K Kit (Zymo Research, USA). Preparation of transposomes was made in a $10 \mu \mathrm{l}$ reaction system as follows: $2.2 \mu \mathrm{g}$ of transposon, $1 \mu \mathrm{l}$ of EZ-Tn5 transposase (Epicentre, USA), and $2.5 \mu \mathrm{l}$ of $100 \%$ glycerol. The reaction was incubated at room temperature for $30 \mathrm{~min}$ and then switched to $4^{\circ} \mathrm{C}$ for another $72 \mathrm{~h}$. In all, $1-2 \mu \mathrm{l}$ of transposomes were transformed into S. islandicus RJW008 $(\triangle \arg D)$ via electroporation as described in ref. ${ }^{37}$. Cell transformation assays were repeated dozens of times in order to collect a sufficient number of transformants to achieve saturation mutagenesis. The theoretical number of transposon insertion colonies was calculated using a derivative of Poisson's law: $N=\ln (1-P) / \ln (1-\mathrm{f}), f=$ average gene size $(900.64 \mathrm{bp}) /$ genome size $(2,586,647 \mathrm{bp})$. To make sure the transposon insertions cover approximately $99.99 \%(P=0.9999)$ of the genome, around 26,448 colonies per library are required. The transformed cells were plated 


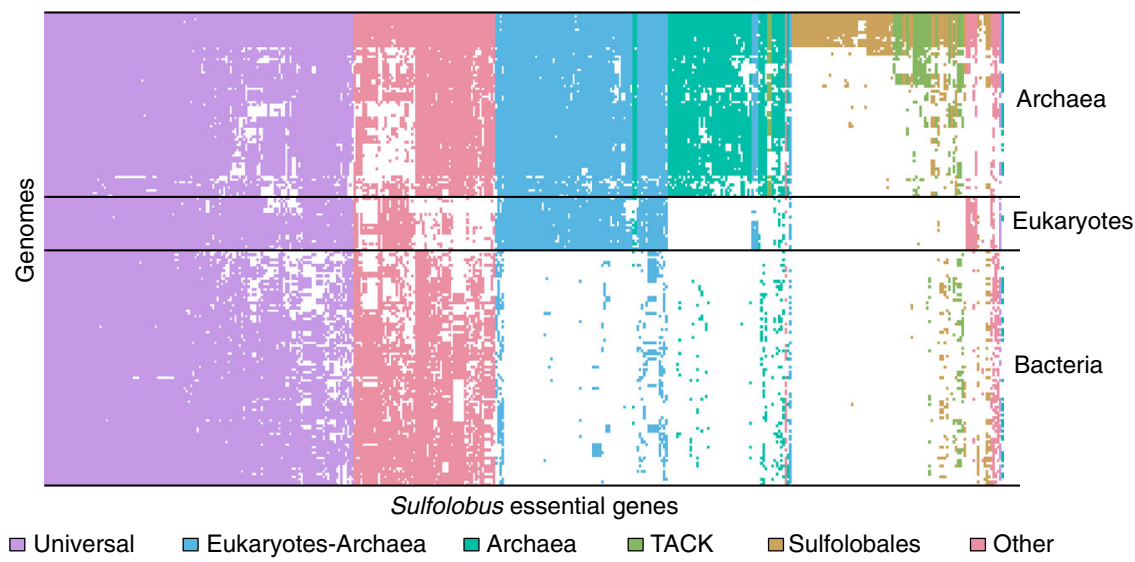

Fig. 6 Presence/absence of genes shows phyletic patterns. Heatmap of shared NOG/arNOGs according to annotations in the eggNOG database corresponding to the essential gene set of S. islandicus M.16.4 across the three domains of life. Each row is 1 of the 177 taxa including the set of 169 used for other distribution analyses, 1 from the candidate phylum Bathyarchaeota, and 7 Asgardarchaeota genomes (Supplementary Data 5 and 6). Each column is 1 of the 441 essential genes discovered in this study. A white box indicates that no matching NOG or arNOG was found, while a colored box indicates presence. Colors indicate categories defined in Tables 1 and 2

Table 2 Number of $S$. islandicus essential genes shared with 168 full genome sequences spanning the tree of life

\begin{tabular}{lllllll} 
& \multicolumn{1}{l}{$\begin{array}{l}\text { Phyletic category } \\
\text { Universal }\end{array}$} & EA & Archaea & TACK & Sulfolobales & Other \\
\hline Shared genes & 141 & 80 & 55 & 18 & 73 & 74 \\
Poorly characterized $^{b}$ & 5 & 1 & 14 & 7 & 46 & 3 \\
\hline
\end{tabular}

aGenes are put into a category if they are present in $\geq 50 \%$ of the organisms in each group, i.e., universal is in $\geq 50 \%$ of each of the Bacteria, Archaea, and Eukarya groups. "Other" refers to genes that do not meet these criteria

bNOG categories "Function unknown" or "General functional prediction only". Full list shown in Supplementary Table 6

on DY plates either by glass beads or over-lay ${ }^{58}$. After 10 days of incubation, the $\mathrm{ArgD}^{+}$revertants were harvested from plates either by manually picking or with sterile spreaders and then pooled into three independent transposon mutant libraries (CYZ-TL1, CYZ-TL2, and CYZ-TL3), with approximately 100,000 colonies in total (Supplementary Table 1). We routinely obtained an average of $c a \cdot 10^{3}$ colonies/ $/ \mathrm{g}$ transposon, and approximately $10^{5}$ colonies/ $\mu \mathrm{g}$ DNA using a replicative plasmid $\mathrm{pSeSd}$-SsoargD. The $\mathrm{pSeSd}$-SsoargD was constructed by cloning the SsoargD marker cassette, amplified from S. solfataricus P2 genomic DNA with primer set SsoargD-F2/R2, into the Xmall site of a Sulfolobus-E.coli shutter vector pSeSd ${ }^{59}$. Thus, the estimated frequency of transposition is $\sim 10^{-2}$ per cell.

\section{DNA library preparation and high-throughput DNA sequencing. Genomic} DNA from each mutant pool was extracted as described in ref. ${ }^{60}$ and then quantified with Qubit ${ }^{\oplus}$ 2.0 Fluorometer (Invitrogen, USA). DNA libraries were prepared using the Nextera XT DNA Library Prep Kit (Illumina, USA) with proper modifications. Briefly, $2 \mathrm{ng}$ of input genomic DNA in total was simultaneously fragmented and tagged with sequencing adapters in a single enzymatic reaction tube. Afterwards, a primer mixture of Tn-seq-F (Supplementary Data 9) and N705 (a randomly selected primer from the Nextera XT DNA Library Prep Kit) was added in the same tube to enrich the transposon-chromosome junction regions via PCR. The PCR conditions were as follows: $72{ }^{\circ} \mathrm{C}$ for $3 \mathrm{~min}, 95^{\circ} \mathrm{C}$ for $30 \mathrm{~s}$, and 22 cycles of denaturation at $95^{\circ} \mathrm{C}$ for $10 \mathrm{~s}$, annealing at $55^{\circ} \mathrm{C}$ for $30 \mathrm{~s}$, and extension at $72{ }^{\circ} \mathrm{C}$ for $30 \mathrm{~s}$. A final extension was performed at $72{ }^{\circ} \mathrm{C}$ for $5 \mathrm{~min}$. The resultant library DNA was cleaned up with AMPure XP beads for three times, eluted in $45-\mu \mathrm{l}$ EB buffer (QIAprep Spin Miniprep Kit, USA), and then quantified with Qubit ${ }^{\circledR} 2.0$ Fluorometer. The final DNA library was quantitated on High-Sensitivity Qubit (Life Technologies) and fragment size was evaluated using the Agilent 2100 Bioanalyzer on a DNA7500 chip (Agilent Technologies), then further quantitated by quantitative PCR on a BioRad CFX Connect Real-Time System (Bio-Rad Laboratories, Inc. CA) to ensure accuracy of quantitation of the library containing properly adapted fragments. The final pool was loaded onto 2 lanes (CYZ_TL1) and 1 lane each (CYZ_TL2 and CYZ_TL3) of a HiSeq 2500 Rapid flowcell for cluster formation and sequencing on an Illumina HiSeq 2500 with Rapid SBS sequencing reagents version 2 . Sequencing by synthesis was performed from one end of the molecules for a total read length of $160 \mathrm{nt}$. The $100 \mu \mathrm{M}$ of custom Read 1 sequencing primer, specific for the Tn-seq-F sequence (Supplementary Data 9), was spiked into the standard Read1 HP10 primer tube (position 18) for sequencing. The run generated.bcl files, which were converted into demultiplexed compressed fastq files using the bcl2fastq v1.8.4 Conversion Software (Illumina, CA) at the W M. Keck Center for Comparative and Functional Genomics at the University of Illinois at Urbana-Champaign.

Tn-seq data processing and analysis. Illumina FASTQ reads from all three libraries that were $<50 \mathrm{bp}$ in length, had a quality score $<30$, and did not contain the 23-bp transposon sequence were removed. The remaining reads were stripped of transposon and adapter sequence and aligned to the S. islandicus M.16.4 genome (NC_012726) using the Burrows-Wheeler Bowtie 2 alignment tool ${ }^{61}$. Reads that mapped to multiple locations in the genome or to ambiguous sites were set aside, as were those with an alignment length $<11 \mathrm{bp}$. Using the in-house software, the resulting .sam alignment files were converted to lists that included unique insertion locations, the strand to which they aligned, and the number of reads associated with that event (Supplementary Data 1). Insertions that occurred in the same location but on different strands or in separate libraries were considered independent events. Tn5 transposase has been shown to prefer certain insertion sites over others ${ }^{62}$, so each reported site was extracted and nucleotide frequency was measured 20 bases upstream and downstream as compared to an equal number of random sites in the genome. Random sampling via the Python numpy.random. choice function (with replacement) yielded sites with overall frequencies matching the known $\mathrm{G}+\mathrm{C}$ content of the genome (35\%), but a pronounced and palindromic pattern was observed at insertion sites even when normalizing for this bias (Supplementary Fig. 9). Overall Tn5 appears to prefer a G-C bp flanked by an AT-rich region, which is consistent with other studies ${ }^{62,63}$. However, when normalized to the overall $\mathrm{G}+\mathrm{C}$ content, no single biased site was more than two-fold enriched in a certain base compared to the rest of the genome, meaning that there was considerable variation in the sites themselves and thus the chance that the bias would significantly affect our results is reduced. Gene essentiality was then evaluated using the software previously designed and published for this purpose: Tn-Seq Explorer $^{24}$ and ESSENTIALS ${ }^{23}$. The ESSENTIALS software was run with mostly default settings with a list of insertion locations and associated reads as the input. The locations for each of the three libraries were submitted as separate files and the total library size specified as 105,968 (Supplementary Table 1). Repeat filtering was enabled to avoid calling repeated regions as essential. The LOESS smoothing feature normally meant to compensate for the over-representation of bacterial origins of replication (caused by multiple simultaneous replication rounds) was disabled because Sulfolobus only undergoes one round of replication per cell cycle ${ }^{64}$. Because of the lack of observed sequence specificity, the insertion site was specified 
as "random." The program uses " $\log _{2} \mathrm{FC}$ " as its measure of essentiality, which is proportional to $\log _{2}$ (reads observed/reads expected) for each gene and sets a cutoff automatically as the local minimum between essential and non-essential distributions in a density plot of the scores. The program suggested a putative maximum $\log _{2} \mathrm{FC}$ of -5.1 for essential genes.

For the Tn-Seq Explorer software, insertion sites of all three libraries were combined and insertion sites with $<4$ reads were excluded for analysis due to their vast over-representation in the insertion sites and the uncertainty of their source (Supplementary Table 1). The program uses a sliding window approach and returns an EI based on the number, location, and spatial concentration of insertion sites within each individual gene. It also allows for the adjustment of the stated start and end points of the gene. As is default, insertions in the first $5 \%$ and last $20 \%$ of genes were excluded to compensate for misannotated start codons and proteins for which C-terminal deletions are tolerated, respectively. The program suggested an EI maximum of 3 (Fig. 1b).

Construction of $\boldsymbol{S}$. islandicus mutants. Except where otherwise stated, disruption of the chromosomal genes was achieved by replacing their coding regions (57-100\% of the length of the gene was deleted) with the $\operatorname{argD}$ expression cassette (StoargD) derived from Sulfolobus tokodaii via a microhomology-mediated gene inactivation approach we recently developed ${ }^{65}$. Briefly, a functional $\arg D$ gene was PCR-amplified from a linearized Sulfolobus-E.coli shuttle vector pSesD-StoargD with 35-40 bp homology of the targeted gene introduced, yielding the gene disruption cassettes. The resultant PCR products were purified and electroporated into the $\operatorname{argD}$ auxotrophic strain $S$. islandicus RJW008, selecting $\operatorname{ArgD}^{+}$transformants on the plates lacking agmatine. S-layer genes $s l a A$, slaB, and $\operatorname{sla} A B$ were deleted from the chromosome of the genetic host S. islandicus RJW004 via an improved MID strategy ${ }^{37,66}$ with knockout plasmids pMID-slaA, pMID-slaB, and pMID-slaAB, respectively. The resulting $\Delta$ sla $A$ and $\Delta$ slaB mutants harbored an inframe deletion of the coding region from nucleotides +52 to +3687 relative to the start codon of slaA ( $3690 \mathrm{bp}$ in length), and +13 to +1185 relative to the start codon of $s l a B$ (1194 bp in length), respectively. The $\triangle$ sla $A B$ mutant was constructed similarly leaving $51 \mathrm{bp}$ of the slaA (nt $1-51$ relative to the start codon of slaA), $6 \mathrm{bp}$ of restriction enzyme (MluI) site, and $9 \mathrm{bp}$ of slaB (nt 1186-1194 relative to the start codon of slaB) in the chromosome of $S$. islandicus RJW004. Verification of each gene replacement or deletion mutant was determined through PCR diagnosis with both flanking primers (bind outside of the targeted region) and internal primers (bind inside of targeted region), which examined the genotype and purity of mutants, respectively. The primers used to generate and confirm gene disruptions or deletions were described in Supplementary Data 9, and the expected sizes of amplicons generated from the genetic host (wt) and mutant strains were provided in Supplementary Table 8.

Transmission electron microscopy. Proteinaceous S-layer was extracted from S. islandicus cell cultures as described in ref. ${ }^{38}$. To prepare the samples that were used for TEM, glow-discharged, carbon-stabilized Formvar-coated 200-mesh copper grids (Carbon Type-B, cat. no. 01811, Ted Pella, Inc., USA) were placed on $8-20-\mu$ d droplets of each sample for $3 \mathrm{~min}$, rinsed with degassed water, and negative-stained with $2 \%$ uranyl acetate for $15-60 \mathrm{~s}$. Thin-sectioned $S$. islandicus cells were prepared essentially as described in ref. ${ }^{67}$, with minor modifications as follows: after microwave fixation with the primary fixative, cells were washed in Sorenson's Phosphate buffer with no further additives. All samples were observed using a Philips CM200 transmission electron microscope at $120 \mathrm{kV}$. Images were taken at various magnifications using a TVIPS (Tietz Video and Image Processing Systems GmbH; Germany) $2 \mathrm{k} \times 2 \mathrm{k}$ Peltier-cooled CCD camera. Scale bars were added with the ImageJ software.

Homology search. Homologs for the 441 essential genes found in Supplementary Data 2 were found across the 168 genomes listed in Supplementary Data 6 via the European Molecular Biology Laboratory eggNOG database ${ }^{51}$. Genomes were downloaded from the National Center for Biotechnology Information (NCBI) (www.ncbi.nlm.nih.gov). The genomes to survey are based on the $\operatorname{set}^{6}$ in Raymann et al. (2015) with the following additions: $M$. maripaludis S2 was added to compare essential gene content; the genome of Toxoplasma gondii ME49 was added because its essential genome became available during the course of this analysis ${ }^{68}$; Schizosaccharomyces pombe ${ }^{69}$ was added to compare with Saccharomyces cerevisiae S288C; additional Sulfolobales genomes were added for intra-order comparison of essential gene content (listed in Supplementary Data 6). While not included in the phyletic distribution analysis, the sequences for Lokiarchaeum sp. GC14_75 $5^{13}$ and Thorarchaeota ${ }^{70}$ SMTZ-45, SMTZ1-45, and SMTZ1-83 were retrieved and analyzed; presence/absence data can be found in Supplementary Data 5. Several bacterial genomes were added to include additional model systems (e.g., E. coli str. K-12 substr. MG1655 and Bacillus subtilis subsp. subtilis str. 168). The complete list is found in Supplementary Data 6. Owing to their incomplete or highly reduced nature, we excluded DPANN and Asgard lineages, as well as Bacteria from the candidate phyla radiation ${ }^{71}$ and the minimal Mycoplasma Syn 3.032; however, presence/absence of information for selected genomes are provided in Supplementary Data 5. For organisms not in the eggNOG database, the amino acid sequences of protein-coding genes were uploaded to the eggNOG mapper tool (http://eggnogdb.embl.de/\#/app/emapper) and run with default settings. These data were translated into a presence/absence matrix and evaluated with custom Python and Zsh scripts to assess the phyletic distribution of essential gene candidates. Finally, for each S. islandicus M.16.4 essential gene candidate, the amino acid sequences of all bidirectional best BLAST hits (BBBH) of that gene across all 168 genomes (BLASTP; minimum 70\% sequence coverage, $20 \%$ identity, $40 \%$ positively scoring sites, and maximum E-value of 0.001 ) were used to scan genomes in which no homologs were found using tBLASTn, and the results were filtered according to the same cutoff criteria as the bidirectional best BLAST hits. The tBLASTn hits that overlapped with annotated genes by $>50 \mathrm{bp}$ were discarded. This was to fill in gaps left by annotation mistakes, where the protein may still be in the genome but was not published as such; however, the approach introduced many false-positives and both bidirecitonal best hits and tBLASTn gene matches often were in disagreement with the eggNOG assignments (data not shown). Due to the better quality of eggNOG matches, neither set of BLAST results were included in presence/absence matrices (data not shown). However, BBBH within selected Sulfolobales can be found in Supplementary Data 2.

Parsimony analysis. Presence/absence matrices were converted to NEXUS format files with a custom Python script and used in the Phylogenetic Analysis Using Parsimony (and other methods) (PAUP*) tool ${ }^{72}$. The main tree was found with the heuristic search function with a maximum of 1000 trees in memory, default settings. The first tree was saved as an unrooted NEXUS format tree with branch lengths. Bootstrapping was run with default settings for 1000 iterations with 100 maximum trees in memory. The resulting tree was saved with support values as node labels. A custom python script using the Phylo package within the Biopython ${ }^{73}$ suite was used to transfer the support values from the bootstrap consensus tree to the corresponding nodes on the heuristic search tree. Trees were visualized in the interactive tree of life $\mathrm{f}^{74}$ interface.

Phyletic distribution analysis. The presence/absence matrices were also crossreferenced with phylogenetic data via NCBI taxonomy information to determine how widespread each gene was in different orders spanning the tree of life. Simulated random distributions of genes were created by counting how many organisms in which they were found and assigning that many random organisms to each gene (without replacement) for each gene 100 times using the numpy. random.choice function. $P$ values were generated by counting the number of simulated observations above or below the true observation and dividing by 100 . To determine the proportion of COGs or arCOGs that have essential members in $S$. islandicus, we removed unique clusters with $>1$ gene in $S$. islandicus that showed no essentiality from the total due to possible redundancy in functional orthologs from the same cluster. To test for bias in the phyletic sampling of the set of 169 genomes, we assembled 100 organism sets with genomes randomly sampled from the eggNOG database to equal proportions of TACK archaea, euryarchaeota, eukaryotes, and bacteria as to that in the 169 genome set. Organisms were chosen at random without replacement using the numpy.random choice function again. In all data involving the eggNOG database, organisms missing from the NOG member file were excluded.

Code availability. The custom Python and Zsh scripts used for analyses in this study are available from the corresponding author upon request.

Reporting summary. Further information on experimental design is available in the Nature Research Reporting Summary linked to this paper.

\section{Data availability}

The raw Tn-seq data of three independent transposon insertion libraries CYZ-TL1, CYZ-TL2, and CYZ-TL3 have been deposited at NCBI under BioSample accession numbers SAMN08628694, SAMN08628695, and SAMN08628696, respectively; Bioproject accession number PRJNA436600; and Sequence Read Archive (SRA) accession number SRP133799. Analyzed data showing the insertion locations across three independent transposon libraries can be found in Supplementary Data 1. All other data that support the findings of this work are available from the corresponding author upon request.

Received: 31 August 2018 Accepted: 24 October 2018 Published online: 21 November 2018

\section{References}

1. Woese, C. R. \& Fox, G. E. Phylogenetic structure of the prokaryotic domain: the primary kingdoms. Proc. Natl Acad. Sci. 74, 5088-5090 (1977).

2. Woese, C. R. \& Fox, G. E. The concept of cellular evolution. J. Mol. Evol. 10, $1-6$ (1977). 
3. Iwabe, N., Kuma, K., Hasegawa, M., Osawa, S. \& Miyata, T. Evolutionary relationship of archaebacteria, eubacteria, and eukaryotes inferred from phylogenetic trees of duplicated genes. Proc. Natl Acad. Sci. 86, 9355-9359 (1989).

4. Woese, C. R., Kandler, O. \& Wheelis, M. L. Towards a natural system of organisms: proposal for the domains Archaea, Bacteria, and Eucarya. Proc. Natl. Acad. Sci. 87, 4576-4579 (1990).

5. Guy, L. \& Ettema, T. J. G. The archaeal 'TACK' superphylum and the origin of eukaryotes. Trends Microbiol. 19, 580-587 (2011).

6. Raymann, K., Brochier-Armanet, C. \& Gribaldo, S. The two-domain tree of life is linked to a new root for the Archaea. Proc. Natl. Acad. Sci. 112, 6670-6675 (2015).

7. Puigbò, P., Wolf, Y. I. \& Koonin, E. V. Search for a 'tree of life' in the thicket of the phylogenetic forest. J. Biol. 8, 59 (2009).

8. Harris, J. K., Kelley, S. T., Spiegelman, G. B. \& Pace, N. R. The genetic core of the universal ancestor. Genome Res. 13, 407-412 (2003).

9. Gil, R. et al. The genome sequence of Blochmannia floridanus: comparative analysis of reduced genomes. Proc. Natl Acad. Sci. 100, 9388-9393 (2003).

10. Weiss, M. C. et al. The physiology and habitat of the last universal common ancestor. Nat. Microbiol. 1, 16116 (2016).

11. Mirkin, B. G., Fenner, T. I., Galperin, M. Y. \& Koonin, E. V. Algorithms for computing parsimonious evolutionary scenarios for genome evolution, the last universal common ancestor and dominance of horizontal gene transfer in the evolution of prokaryotes. BMC Evol. Biol. 3, 2 (2003).

12. Makarova, K., Wolf, Y. \& Koonin, E. Archaeal clusters of orthologous genes (arCOGs): an update and application for analysis of shared features between Thermococcales, Methanococcales, and Methanobacteriales. Life 5, 818-840 (2015).

13. Spang, A. et al. Complex archaea that bridge the gap between prokaryotes and eukaryotes. Nature 521, 173-179 (2015).

14. Zaremba-Niedzwiedzka, K. et al. Asgard archaea illuminate the origin of eukaryotic cellular complexity. Nature 541, 353-358 (2017).

15. Cunha, V. D., Gaia, M., Gadelle, D., Nasir, A. \& Forterre, P. Lokiarchaea are close relatives of Euryarchaeota, not bridging the gap between prokaryotes and eukaryotes. PLoS Genet. 13, e1006810 (2017).

16. Spang, A. et al. Asgard archaea are the closest prokaryotic relatives of eukaryotes. PLoS Genet. 14, e1007080 (2018).

17. Cunha, V. D., Gaia, M., Nasir, A. \& Forterre, P. Asgard archaea do not close the debate about the universal tree of life topology. PLoS Genet. 14, e1007215 (2018).

18. Rivera, M. C. \& Lake, J. A. The ring of life provides evidence for a genome fusion origin of eukaryotes. Nature 431, 152 (2004).

19. Eme, L., Spang, A., Lombard, J., Stairs, C. W. \& Ettema, T. J. G. Archaea and the origin of eukaryotes. Nat. Rev. Microbiol. 15, 133 (2017).

20. Leigh, J. A., Albers, S.-V., Atomi, H. \& Allers, T. Model organisms for genetics in the domain Archaea: methanogens, halophiles, Thermococcales and Sulfolobales. FEMS Microbiol. Rev. 35, 577-608 (2011).

21. Peng, N., Han, W., Li, Y., Liang, Y. \& She, Q. Genetic technologies for extremely thermophilic microorganisms of Sulfolobus, the only genetically tractable genus of crenarchaea. Sci. China Life Sci. 60, 370-385 (2017).

22. Albers, S.-V. \& Meyer, B. H. The archaeal cell envelope. Nat. Rev. Microbiol. 9, 414-426 (2011).

23. Zomer, A., Burghout, P., Bootsma, H. J., Hermans, P. W. M. \& van Hijum, S. A. F. T. ESSENTIALS: software for rapid analysis of high throughput transposon insertion sequencing data. PLoS ONE 7, e43012 (2012).

24. Solaimanpour, S., Sarmiento, F. \& Mrázek, J. Tn-Seq explorer: a tool for analysis of high-throughput sequencing data of transposon mutant libraries. PLoS ONE 10, e0126070 (2015).

25. Zhang, C., She, Q., Bi, H. \& Whitaker, R. J. The apt/6-methylpurine counterselection system and its applications in genetic studies of the hyperthermophilic archaeon Sulfolobus islandicus. Appl. Environ. Microbiol. 82, 3070-3081 (2016).

26. Zhang, C. et al. Genetic manipulation in Sulfolobus islandicus and functional analysis of DNA repair genes. Biochem. Soc. Trans. 41, 405-410 (2013).

27. Song, X., Huang, Q., Ni, J., Yu, Y. \& Shen, Y. Knockout and functional analysis of two DExD/H-box family helicase genes in Sulfolobus islandicus REY15A. Extremophiles 20, 537-546 (2016).

28. Liu, J. et al. Functional assignment of multiple ESCRT-III homologs in cell division and budding in Sulfolobus islandicus. Mol. Microbiol. 105, 540-553 (2017).

29. Hong, Y. et al. Dissection of the functional domains of an archaeal Holliday junction helicase. DNA Repair (Amst.) 11, 102-111 (2012).

30. Luo, H., Lin, Y., Gao, F., Zhang, C.-T. \& Zhang, R. DEG 10, an update of the database of essential genes that includes both protein-coding genes and noncoding genomic elements. Nucleic Acids Res. 42, D574-D580 (2014).

31. Sarmiento, F., Mrazek, J. \& Whitman, W. B. Genome-scale analysis of gene function in the hydrogenotrophic methanogenic archaeon Methanococcus maripaludis. Proc. Natl Acad. Sci. 110, 4726-4731 (2013).
32. Hutchison, C. A. et al. Design and synthesis of a minimal bacterial genome. Science 351, aad6253 (2016)

33. Taylor, K. A., Deatherage, J. F. \& Amos, L. A. Structure of the S-layer of Sulfolobus acidocaldarius. Nature 299, 840 (1982).

34. Baumeister, W., Wildhaber, I. \& Phipps, B. M. Principles of organization in eubacterial and archaebacterial surface proteins. Can. J. Microbiol. 35, 215-227 (1989).

35. Veith, A. et al. Acidianus, Sulfolobus and Metallosphaera surface layers: structure, composition and gene expression. Mol. Microbiol. 73, 58-72 (2009).

36. Rodrigues-Oliveira, T., Belmok, A., Vasconcellos, D., Schuster, B. \& Kyaw, C. M. Archaeal S-layers: overview and current state of the art. Front. Microbiol. 8, 2597 (2017).

37. Zhang, C., Cooper, T. E., Krause, D. J. \& Whitaker, R. J. Augmenting the genetic toolbox for Sulfolobus islandicus with a stringent positive selectable marker for agmatine prototrophy. Appl. Environ. Microbiol. 79, 5539-5549 (2013).

38. Peyfoon, E. et al. The S-layer glycoprotein of the Crenarchaeote Sulfolobus acidocaldarius is glycosylated at multiple sites with chitobiose-linked $\mathrm{N}$ glycans. Archaea 2010, 754101 (2010).

39. Reitz, T. et al. Spectroscopic study on uranyl carboxylate complexes formed at the surface layer of Sulfolobus acidocaldarius. Dalton Trans. 44, 2684-2692 (2015).

40. Forterre, P. A hot story from comparative genomics: reverse gyrase is the only hyperthermophile-specific protein. Trends Genet. 18, 236-237 (2002).

41. Brochier-Armanet, C. \& Forterre, P. Widespread distribution of archaeal reverse gyrase in thermophilic bacteria suggests a complex history of vertical inheritance and lateral gene transfers. Archaea 2, 83-93 (2006).

42. Han, W., Feng, X. \& She, Q. Reverse gyrase functions in genome integrity maintenance by protecting DNA breaks in vivo. Int J. Mol. Sci. 18, 1340 (2017).

43. Lintner, N. G. et al. Structural and functional characterization of an archaeal clustered regularly interspaced short palindromic repeat (CRISPR)-associated complex for antiviral defense (CASCADE). J. Biol. Chem. 286, 21643-21656 (2011).

44. Zhang, R. \& Lin, Y. DEG 5.0, a database of essential genes in both prokaryotes and eukaryotes. Nucleic Acids Res. 37, D455-D458 (2009).

45. Giaever, G. et al. Functional profiling of the Saccharomyces cerevisiae genome. Nature 418, 387-391 (2002)

46. Kim, D.-U. et al. Analysis of a genome-wide set of gene deletions in the fission yeast Schizosaccharomyces pombe. Nat. Biotechnol. 28, 617-623 (2010).

47. Veeranagouda, Y., Husain, F., Tenorio, E. L. \& Wexler, H. M. Identification of genes required for the survival of $B$. fragilis using massive parallel sequencing of a saturated transposon mutant library. BMC Genomics 15, 429 (2014).

48. Kobayashi, K. et al. Essential Bacillus subtilis genes. Proc. Natl Acad. Sci. 100, 4678-4683 (2003).

49. Commichau, M., F., Pietack, N. \& Stülke, J. Essential genes in Bacillus subtilis: a re-evaluation after ten years. Mol. Biosyst. 9, 1068-1075 (2013).

50. Woese, C. R. On the evolution of cells. Proc. Natl Acad. Sci. 99, 8742-8747 (2002).

51. Huerta-Cepas, J. et al. eggNOG 4.5: a hierarchical orthology framework with improved functional annotations for eukaryotic, prokaryotic and viral sequences. Nucleic Acids Res. 44, D286-D293 (2016).

52. Ciccarelli, F. D. et al. Toward automatic reconstruction of a highly resolved tree of life. Science 311, 1283 (2006).

53. Galcheva-Gargova, Z., Konstantinov, K. N., Wu, I.-H. \& Klier, F. G. Binding of zinc finger protein ZPR1 to the epidermal growth factor receptor. Science 272, 1797 (1996).

54. Gangwani, L., Mikrut, M., Galcheva-Gargova, Z. \& Davis, R. J. Interaction of ZPR1 with translation elongation factor-1 $\alpha$ in proliferating cells. J. Cell Biol. 143, 1471-1484 (1998).

55. Gangwani, L. Deficiency of the zinc finger protein ZPR1 causes defects in transcription and cell cycle progression. J. Biol. Chem. 281, 40330-40340 (2006).

56. Mishra, A. K., Gangwani, L., Davis, R. J. \& Lambright, D. G. Structural insights into the interaction of the evolutionarily conserved ZPR1 domain tandem with eukaryotic EF1A, receptors, and SMN complexes. Proc. Natl Acad. Sci. 104, 13930-13935 (2007).

57. Kiełbowicz-Matuk, A., Czarnecka, J., Banachowicz, E., Rey, P. \& Rorat, T. Solanum tuberosum ZPR1 encodes a light-regulated nuclear DNA-binding protein adjusting the circadian expression of StBBX24 to light cycle. Plant Cell Environ. 40, 424-440 (2017).

58. Deng, L., Zhu, H., Chen, Z., Liang, Y. X. \& She, Q. Unmarked gene deletion and host-vector system for the hyperthermophilic crenarchaeon Sulfolobus islandicus. Extremophiles 13, 735 (2009).

59. Peng, N. et al. A synthetic arabinose-inducible promoter confers high levels of recombinant protein expression in hyperthermophilic archaeon Sulfolobus islandicus. Appl. Environ. Microbiol. 78, 5630-5637 (2012). 
60. Reno, M. L., Held, N. L., Fields, C. J., Burke, P. V. \& Whitaker, R. J. Biogeography of the Sulfolobus islandicus pan-genome. Proc. Natl Acad. Sci. 106, 8605-8610 (2009).

61. Langmead, B. \& Salzberg, S. L. Fast gapped-read alignment with Bowtie 2. Nat. Methods 9, 357-359 (2012).

62. Green, B., Bouchier, C., Fairhead, C., Craig, N. L. \& Cormack, B. P. Insertion site preference of $\mathrm{Mu}, \mathrm{Tn} 5$, and Tn7 transposons. Mob. DNA 3, 3 (2012).

63. Reznikoff, W. S. The Tn5 transposon. Annu Rev. Microbiol. 47, 945-964 (1993).

64. Bernander, R. \& Poplawski, A. Cell cycle characteristics of thermophilic archaea. J. Bacteriol. 179, 4963-4969 (1997).

65. Zhang, C. \& Whitaker, R. J. Microhomology-mediated high-throughput gene inactivation strategy for the hyperthermophilic crenarchaeon Sulfolobus islandicus. Appl. Environ. Microbiol. 84, e02167-17 (2018).

66. Zhang, C. et al. Revealing the essentiality of multiple archaeal PCNA genes using a mutant propagation assay based on an improved knockout method. Microbiology 156, 3386-3397 (2010).

67. Bautista, M. A., Zhang, C. \& Whitaker, R. J. Virus-induced dormancy in the archaeon Sulfolobus islandicus. mBio 6, e02565-14 (2015).

68. Sidik, S. M. et al. A genome-wide CRISPR screen in toxoplasma identifies essential apicomplexan genes. Cell 166, 1423.e12-1435.e12 (2016).

69. Wood, V. et al. The genome sequence of Schizosaccharomyces pombe. Nature 415, 871 (2002).

70. Seitz, K. W., Lazar, C. S., Hinrichs, K.-U., Teske, A. P. \& Baker, B. J. Genomic reconstruction of a novel, deeply branched sediment archaeal phylum with pathways for acetogenesis and sulfur reduction. ISME J. 10, 1696-1705 (2016).

71. Hug, L. A. et al. A new view of the tree of life. Nat. Microbiol. 1, 16048 (2016).

72. Swofford, D. L. PAUP*. Phylogenetic Analysis using Parsimony ( ${ }^{*}$ and Other Methods). Version 4 (Sinauer Associates, Sunderland, MA, 2003).

73. Cock, P. J. A. et al. Biopython: freely available Python tools for computational molecular biology and bioinformatics. Bioinformatics 25, 1422-1423 (2009).

74. Letunic, I. \& Bork, P. Interactive tree of life (iTOL) v3: an online tool for the display and annotation of phylogenetic and other trees. Nucleic Acids Res. 44, W242-W245 (2016).

\section{Acknowledgements}

We thank Chris L. Wright and Alvaro G. Hernandez from W.M. Keck Center for Comparative and Functional Genomics, University of Illinois at Urbana-Champaign (UIUC) for advice with primer design, DNA library construction, and sequencing. We also thank Whitney E. England, Angelo Blancaf, and Ted Kim for assistance in Tn-seq data processing; Carlos A. Vega, Elizabeth H. Marr, and Melinda E. Baughman for providing technical assistance in collecting transposon insertion colonies. We thank Isaac K.O. Cann and Scott C. Dawson for fruitful discussions. We are thankful to Kira S. Makarova for technical assistance with data retrieval from the arCOG database and for helpful suggestions. We thank Marleen van Wolferen for providing the S-layer extraction protocol. We acknowledge Yuan Li and Emily N. Hallett for providing S-layer extraction assistance. We would also like to thank Scott J. Robinson from Beckman Institute for Advance Science and Technology, UIUC for technical assistance with
TEM imaging and sample preparation. We thank Lou A. Miller from Frederick Seitz Materials Research Laboratory Central Research Facilities, UIUC for preparing the thin-sectioned $S$. islandicus cells. Funding for this work was mainly provided by the National Aeronautics and Space Administration (NASA) through the NASA Astrobiology Institute under cooperative agreement no. NNA13AA91A, issued through the Science Mission Directorate. This work was also partially supported by Division of Environmental Biology (DEB: 1355171 to R.J.W.), US National Science Foundation, the Department of Microbiology Alice Helm Graduate Research Excellence Fellowship, UIUC (to A.P.R.P.), the Carl R. Woese Institute for Genomic Biology Undergraduate Research Scholar program, and the Office of Undergraduate Research, UIUC (to R.L.W).

\section{Author contributions}

C.Z., A.P.R.P. and R.J.W. conceived and designed the research; C.Z. and R.L.W. carried out experimental work; A.P.R.P., C.Z., G.J.O., R.L.W. and R.J.W. analyzed the data; R.J.W. and G.J.O contributed new reagents/analytic tools; and C.Z., A.P.R.P., R.L.W. and R.J.W. wrote the paper. All authors edited the manuscript.

\section{Additional information}

Supplementary Information accompanies this paper at https://doi.org/10.1038/s41467018-07379-4.

Competing interests: The authors declare no competing interests.

Reprints and permission information is available online at http://npg.nature.com/ reprintsandpermissions/

Publisher's note: Springer Nature remains neutral with regard to jurisdictional claims in published maps and institutional affiliations.

\begin{abstract}
Open Access This article is licensed under a Creative Commons
Attribution 4.0 International License, which permits use, sharing,
adaptation, distribution and reproduction in any medium or format, as long as you give (c) Open Access This article is licensed under a Creative Commons
Attribution 4.0 International License, which permits use, sharing,
adaptation, distribution and reproduction in any medium or format, as long as you give appropriate credit to the original author(s) and the source, provide a link to the Creative Commons license, and indicate if changes were made. The images or other third party material in this article are included in the article's Creative Commons license, unless indicated otherwise in a credit line to the material. If material is not included in the article's Creative Commons license and your intended use is not permitted by statutory regulation or exceeds the permitted use, you will need to obtain permission directly from the copyright holder. To view a copy of this license, visit http://creativecommons.org/ licenses/by/4.0/.
\end{abstract}

(c) The Author(s) 2018 